\title{
Effective Constraint based Clustering Approach for Collaborative Filtering Recommendation using Social Network Analysis
}

S. Kanimozhi profiles are most similar to that of user $u$. Content-based methods use the features of items, e.g. movie's genres, directors, actors, etc., to generate recommendations. Hybrid approaches [4] make recommendations by combining collaborative filtering and content-based recommendation.

Collaborative Filtering (CF) [14] is a widely used technique in recommender systems. The other is collaborative filtering (CF), which finds their wanted items by making recommendations based on the ratings of similar users on the recommended items. Since the CF is proposed, it has proved to be one of the most successful techniques in recommender systems by its advantage of that no explicit description of items is needed.

Methods in CF can be either memory-based or modelbased. Memory-based algorithms operate on the entire useritem rating matrix and generate recommendations by identifying the neighborhood of the target user to whom the recommendations will be made, based on the agreement of user's past ratings. Model-based techniques use the rating data to train a model and then the model will be used to derive the recommendations. Well-known model-based techniques include clustering [15], pLSA [10], matrix factorization (e.g. SVD) [13], machine learning on the graph [16], etc. Memorybased techniques are quite successful in real-world applications because they are easy to understand, easy to implement and work well in many real-world situations. However, there are some problems which limit the application of memory-based techniques, especially in the large-scale applications like Amazon.com. The most serious problem is the sparsity of user-item rating matrix where each user only rates a small set of a large database of items. The similarity between users (or items) is often derived from few overlapping ratings and it is hence a noisy and unreliable value. Another problem of memory-based CF is efficiency. It has to compute the similarity between every pair of users (or items) to determine their neighborhoods. This is not computationally feasible for the ad-hoc recommender systems with millions of users and items.

To overcome the weaknesses of memory-based techniques, a line of research has focussed on model-based clustering techniques with the aim of seeking more accurate, yet more efficient methods. Based on ratings, these techniques group users or items into clusters, thus give a new way to identify the neighborhood. In this paper, clustering techniques using social information is focused.

S. Kanimozhi, Gobi Arts and Science College, Gobichettipalayam, Email: kanipuppy.it@gmail.com 
Clustering [2] is one of the most popular approaches used for data analysis and classification. Cluster analysis is widely used in disciplines that involve analysis of multivariate data. A search through Google Scholar found 1,660 entries with the words data clustering that comes into sight in 2007 alone. This huge amount of data provides the significance of clustering in data analysis. It is very complex to list the different scientific fields and applications that have utilized clustering method as well as the thousands of existing techniques.

The main objective is to identify the communities of similar users based on their social relationships and use these communities as a mechanism to make the recommendations. Currently, some recommender systems allow users to build their social networks and use this network as additional information to suggest items to users.

\section{RELATED WORKS}

Collaborative filtering recommendation algorithm has proved to be one of the most successful algorithms in recommender systems in recent years. However, traditional centralized collaborative filtering system has suffered from its shortage in scalability as their calculation complexity increases quickly both in time and space when the number of the user and item in the rating database increases. As a result, distributed collaborative filtering is attracting increasing attention as an alternative implementation scheme for collaborative filtering recommender systems. This paper by SongJie et al., [7] proposes a distributed collaborative filtering architecture based on peer-to-peer networks, because of its advantage of scalability as an alternative architecture. The peers communicate each other by sending messages. The peerto peer application advantage is employed in order to manage the user-item rating database, produce the prediction and top$\mathrm{N}$ recommendation.

In a service-oriented online social network consisting of service providers and consumers, a service consumer can search trustworthy service providers via the social network. This requires the evaluation of the trustworthiness of a service provider along a certain social trust path from the service consumer to the service provider. However, there are usually many social trust paths between participants in social networks. Thus, a challenging problem is which social trust path is the optimal one that can yield the most trustworthy evaluation result. In this paper, Guanfeng et al., [11] first present a novel complex social network structure and a new concept, Quality of Trust (QoT). The author then modeled the optimal social trust path selection with multiple end-to-end QoT constraints as a Multi-Constrained Optimal Path (MCOP) selection problem which is NP-Complete. For solving this challenging problem, we propose an efficient heuristic algorithm, H OSTP. The results of our experiments conducted on a large real dataset of online social networks illustrate that our proposed algorithm significantly outperforms existing approaches.

Inspired by the recent successes of boosting algorithms, a trend in unsupervised learning has begun to emphasize the need to explore the design of weighted clustering algorithms. In this paper, we handle clustering as a constrained minimization of a Bregman divergence. Theoretical results show benefits resembling those of boosting algorithms, and bring new modified weighted versions of clustering algorithms such as k-means, expectation-maximization (EM) and kharmonic means. Experiments display the quality of the results obtained, and corroborate the advantages that subtle data reweightings may indeed bring to clustering.

\section{Memory-BASED Collaborative Filtering}

Memory-based CF (user-based or item-based) is based on the fact that users often like the items which are preferred by others users who have agreed with them in the past. Memorybased $\mathrm{CF}$ uses the entire user-item rating database to generate recommendations. A typical CF algorithm proceeds in three steps:

1. Calculating the similarity, wi,j, between active user/item $i$ and user/item $j$.

2. Neighborhood formation: select $k$ similar users/items.

3. Generating top $N$ items by weighted average of all the ratings of users/items in the neighborhood.

\subsection{Similarity Computation}

Similarity computation is a crucial step of CF algorithms. The basic idea of similarity computation is co-rating. For userbased CF, similarity between user $i$ and user $j$ is computed using the items which have been rated by both users. For itembased CF, similarity between item $i$ and item $j$ is computed by working on the users who have rated both of these items. Among many methods to compute similarity, Pearson correlation and vector Cosine are most popular and widely used. For user-based CF, Pearson correlation between user $u$ and user $v$ is computed as follows:

$$
w_{u, v}=\frac{\sum_{i \in I}\left(r_{u, i}-\bar{r}_{u}\right)\left(r_{v, i}-\bar{r}_{v}\right)}{\sqrt{\sum_{i \in I}\left(r_{u, i}-\bar{r}_{u}\right)^{2}} \sqrt{\sum_{i \in I}\left(r_{v, i}-\bar{r}_{v}\right)^{2}}}
$$

where $I$ is the set of items rated by both user $i$ and user $j, r u, i$ is the rating of user $u$ on item $i$ and $r i$ is the average rating of user $i$.

For item-based CF, Pearson correlation between user $i$ and user $j$ is computed as follows:

$$
w_{i, j}=\frac{\sum_{u \in U}\left(r_{u, i}-\bar{r}_{i}\right)\left(r_{u, j}-\bar{r}_{j}\right)}{\sqrt{\sum_{u \in U}\left(r_{u, i}-\bar{r}_{i}\right)^{2}} \sqrt{\sum_{u \in U}\left(r_{u, j}-\bar{r}_{j}\right)^{2}}}
$$

where $U$ is the set of users who have rated both item $i$ and item $j, r_{u, i}$ is the rating of user $u$ on item $i$ and $r_{i}$ is the average rating of item $i$.

Vector Cosine similarity is computed by treating each user as a vector of ratings of the user on items and measuring the cosine of the angle formed by these vectors. Formally, if $R$ is the $m \times n$ user-item rating matrix, then the similarity between 
two users $u$ and $v$ is defined as the cosine of the $m$ dimensional vectors corresponding to the $u$ th and $v$ th row of the matrix $R$.

$$
w_{u, v}=\cos (u, v)=\frac{\vec{u} \cdot \vec{v}}{\|\vec{\imath}\| *\|\vec{\imath}\|}
$$

where $\cdot$ denotes the dot-product of the two vectors. The cosine similarity between two items is computed analogously where $u$ and $v$ correspond to the $u t h$ and $v$ th column of the matrix $R$.

\subsection{Neighborhood Selection}

After the similarity computation, CF algorithms have to select the most similar users for the active user. This is the important step since the recommendations are generated using the ratings of neighbors and therefore neighborhood has an impact on the recommendation quality. According to [17], there are five strategies for neighbor selection:

1. Baseline strategy: select the top $k$ nearest-neighbors who have rated the given item.

2. Baseline strategy with overlap threshold: select the top $k$ nearest-neighbors who have rated the given item and who have rated at least $\phi$ items as the active user has rated (overlapped with the active user).

3. Similarity strategy: select the top nearest-neighbors purely according to their similarities with the active user.

4. Combination strategy: a combination of the strategy 1 and strategy 3.

5. Combination strategy with overlap threshold: a combination of strategies 1,2 and 3 .

A neighborhood selection strategy is chosen depending on the similarity measures and the application domains. In the actual situations, users may use different rating scales, which Cosine similarity cannot take into account. However, using Pearson we also have problems, where some of the neighbors might have only few common rating with the active user. But they could have high similarity value with the active user by chance. The reason is that Pearson correlation does not take into account the degree of overlaps between users. If we use, for example, the first strategy then it could exclude many high similar neighbors just because they have not rated the given items. The combination of these strategies

is preferable, but it could decrease the performance of the algorithms.

\subsection{Generating Recommendation}

In the user-based algorithms, when a subset of nearest neighbors (neighborhood) of the active user are chosen, predictions are generated based on a weighted aggregate of their ratings. Most used aggregating functions are weighted sum and simple weighted average. To make the prediction for the active user $a$ on an

item $i$, weighted sum is computed using all the ratings of the neighbors on that item by the following formula:

$$
P_{a, j}=\bar{r}_{a}+\frac{\sum_{u \in U}\left(r_{u, i}-\bar{r}_{u}\right) w_{a, u}}{\sum_{u \in U}\left\|w_{a, u}\right\|}
$$

Using simple weighted average, the prediction can also be computed by the following formula:

$$
P_{a, j}=\frac{\sum_{u \in U} r_{u, i} w_{a, u}}{\sum_{u \in U}\left\|w_{a, u}\right\|}
$$

Where $\bar{r}_{a}$ and $\bar{r}_{u}$ are the average ratings of user $a$ and user $u$, $w_{a, u}$ is the weight between user $a$ and user $u$. The summations are over all the users $u \in U$ who have rated item $i$. The prediction is generated analogously in item-based algorithms where the summations are over all the ratings of user $a$ on the items in the neighborhood of item $i$ and the weight is the similarity between item $i$ and the items in its neighborhood.

\section{Network Clustering For CF}

The application of clustering techniques reduces the sparsity and improves the scalability of the systems since the similarity can be calculated only for users in the same clusters. Different clustering strategies can be performed based on users and items, as illustrated in Fig. 1. In general, clustering users (or items) results in creating sub-matrices of the entire user-item rating matrix. Then classical CF algorithms (userbased and item-based) can be used to generate recommendation based on these sub-matrices.

In Fig. 1(a), when user-based CF is applied on user clusters, the neighbors of the active user are users in the same user cluster. If item-based CF is used, the ranking of an item is based on the items which are

rated by users in the same user cluster. Similarly, in Fig. 1(b), the neighbors of the active user are users who have rated items in the same item cluster (in case of user-based CF) and the ranking of an item is based on the items in the same item cluster (in case of item-based CF). Fig. 1 depicts the situation where each user and each item are assigned uniquely to one cluster, though users can be assigned to different user clusters (in item clustering) and items can belong to different item clusters (in user clustering). In this case, the prediction for the active user can be made by averaging the opinions of the others in the same user cluster (user-based) and the ranking of an item is based on the items in the same item cluster (itembased). However, in a real-world application, users and items can belong to several clusters, e.g. one user may be interested in the movies of different genres such as horror, war, comedy or drama. One movie could also be assigned to different categories according to genre. The prediction is then made by an average across the clusters.

One simple clustering technique is to classify items based on their content, e.g. movies are categorized by genre. Then the prediction for an item is made by an average of the opinions of users in the clusters that this item belongs to. However, this technique is not feasible in the domains where 
the features of the items are hidden or hard to extract, as well as where the structure of the categories is complicated, for example when items are hierarchically classified. Consequently, several approaches have been proposed to cluster items based on user rating.

\subsection{Algorithm}

The social relationships have an impact on user behavior in recommender systems and propose to use clustering technique on social network of users to identify their neighborhood. We present the first case as depicted in Fig. 1(a), where user-based $\mathrm{CF}$ is combined with user-based clustering. It differs from the traditional model-based CF clustering in two folds. On the one hand, it exploits the social relationship of users, while others techniques cluster users based on the ratings. On the other hand, the clusters are extracted from the network topology, which are quite different to the explicit communities studied in [8] and [3].

Traditional CF algorithms proceed in two phases. At the first phase, calculate the similarities between pairs of users and identify their neighborhood. Then recommendations are generated for active user based on the aggregate of the ratings of the neighbors. In our approach, first we cluster users (offline). Then we apply traditional CF process within clusters to generate recommendations. The algorithm is as follows:

1. Formulate the social network of users, $G=(U, E)$, where $U$ is the set of users, $U=\left\{u_{1}, u_{2}, \ldots, u_{n}\right\}$ and $E$ is the set of social relations between users. $G$ might be a weighted or un-weighted network.

2. Perform a clustering algorithm on the network $G$. The set of users $U$ is divided into clusters $U_{1} \cup U_{2} \ldots \cup U_{q}$, where $V i \cap U j=\emptyset$ and $U=U_{1} \cup U_{2} \ldots \cup U_{q}$.

3. Examine each point in the data set and assign it to the cluster when the violate-constraints ( ) returns false

4. Using clusters as the neighborhoods, the prediction rating for the active user $u$ on item $i$ is computed by either weighted sum or simple weighted average:

$$
\begin{gathered}
P_{a, j}=\bar{r}_{a}+\frac{\sum_{u \in U}\left(r_{u, i}-\bar{r}_{u}\right) w_{a, u}}{\sum_{u \in U}\left\|w_{a, u}\right\|} \\
P_{a, j}=\frac{\sum_{u \in U} r_{u, i} w_{a, u}}{\sum_{u \in U}\left\|w_{a, u}\right\|}
\end{gathered}
$$

where $P_{a, j}$ is the prediction rating of user $a$ for item $i, \bar{r}_{a}$ is the average rating of user $a, U_{a}$ is the cluster of user $a$ and $\mathrm{w}(a, u)$ is the weight between user $a$ and user $u$. The weight $\mathrm{w}(a, u)$ could be the similarity between active user $a$ and the user $u$ in the same cluster, computed using the ratings on the items rated by users in that cluster. That results in a pure memory-based $\mathrm{CF}$ with clustering. The weight $\mathrm{w}(a, u)$ could also be computed based on the social information, for example the (directed/indirected) trust value that user $a$ gives to user $u$, resulting in a social recommendation with clustering. When $\mathrm{w}(a, u)$ is set to 1 , we have group recommendation where every user in a cluster gets the same recommendation for a given item.

\section{Function violate-constraints ()}

if must_link constraint not satisfied return true

elseif cannot_link constraint not satisfied return true

elseif $\delta$-constraint not satisfied return true

elseif $\varepsilon$-constraint not satisfied return true

else return false

Constraints used

The Constraints $[11,12,13]$ used in this paper for Clustering are

- Must-link constraint

- Cannot-link constraint

- $\delta$-constraint

- $\varepsilon$-constraint

Consider $\mathrm{U}=\left\{\mathrm{u}_{1}, \mathrm{u}_{2}, \ldots, \mathrm{u}_{\mathrm{n}}\right\}$ as a set of $\mathrm{n}$ data points that are to be separated into clusters. For any pair of points $U_{i}$ and $\mathrm{U}_{\mathrm{j}}$ in $\mathrm{U}$, the distance between them is represented by $d\left(\mathrm{u}_{\mathrm{i}}, \mathrm{u}_{\mathrm{j}}\right)$ with a symmetric property in order that $d\left(u_{i}, u_{j}\right)=d\left(u_{j}, u_{i}\right)$. The constraints are:

- Must-link constraints indicates that two points $\mathrm{u}_{\mathrm{i}}$ and $\mathrm{u}_{\mathrm{j}}(\mathrm{i} \neq \mathrm{j})$ in $\mathrm{S}$ have to be in the same cluster.

- Cannot-link constraints indicates that two point $\mathrm{u}_{\mathrm{i}}$ and $\mathrm{u}_{\mathrm{j}}(\mathrm{i} \neq \mathrm{j})$ in $\mathrm{S}$ must not be placed in the same cluster.

- $\delta$-Constraint: This constraint represents a value $\delta>0$. Properly, for any pair of clusters $u_{i}$ and $u_{j}(i \neq j)$, and any pair of points $\mathrm{u}_{\mathrm{p}}$ and $\mathrm{u}_{\mathrm{q}}$ such that $\mathrm{u}_{\mathrm{p}} \in \mathrm{S}_{\mathrm{i}}$ and $\mathrm{u}_{\mathrm{q}} \in$ $\mathrm{U}_{\mathrm{j}}, \mathrm{d}\left(\mathrm{u}_{\mathrm{p}}, \mathrm{u}_{\mathrm{q}}\right) \geq \delta$.

- $\quad \varepsilon$-Constraint: This constraint represents a value $\varepsilon>0$ and the feasibility need is the following: for any cluster $S_{i}$ containing two or more points and for any point $\mathrm{u}_{\mathrm{p}} \in \mathrm{U}_{\mathrm{i}}$, there must be another point $\mathrm{u}_{\mathrm{q}} \in \mathrm{U}_{\mathrm{i}}$ such that $\mathrm{d}\left(\mathrm{u}_{\mathrm{p}}, \mathrm{u}_{\mathrm{q}}\right) \leq \varepsilon$.

Must-link constraint and Cannot-link constraint are determined with the help of appropriate neural network. For this purpose, this paper uses Self Organizing Map.

\subsection{Self Organizing Map}

Self-Organizing Maps (SOM) is a general type of neural network technique that is nonlinear regression method that can be utilized to determine relationships amond inputs and outputs or categorize data so as to reveal so far unidentified patterns or structures. It is an outstanding technique in exploratory phase of data mining. The results of the examination represents that self-organizing maps can be a 
feasible technique for categorization of large quantity of data. The SOM has set up its place as an expensively used technique in data-analysis and visualization of highdimensional data. Among other statistical technique the SOM has no close counterpart, and thus it offers a balancing sight to the data. On the other hand, SOM is the most extensively used technique in this group as it offers some notable merits among the substitutes. These comprise, ease of use, particularly for inexperienced users, and highly intuitive display of the data anticipated on to a regular two-dimensional slab, as on a sheet of a paper. The most important prospective of the SOM is in exploratory data analysis that varies from regular statistical data analysis in that there are no assumed set of hypotheses that are validated in the analysis. As an alternative, the hypotheses are created from the data in the data-driven exploratory stage and validated in the confirmatory stage. There are few demerits where the exploratory stage may be adequate alone, such as visualization of data with no additional quantitative statistical inference upon it. In practical data analysis problems the majority of mission is to identify dependencies among variables. In such a difficulty, SOM can be utilized for getting insight to the data and for the original search of potential dependencies. In general the findings require to be validated with more conventional techniques, for the purpose of assessing the assurance of the conclusions and to discard those that are not statistically important.

Initially the chosen parameters are normalized and then initialize the SOM network. Then SOM is trained to offer the maximum likelihood estimation, so that an exacting stock can be linked with a particular node in the categorization layer. The self-organizing networks suppose a topological structure between the cluster units. There are $\mathrm{m}$ cluster units, prearranged in a one or two dimensional array: the input signals are $\mathrm{n}$ dimensional. Figure 1 represents architecture of self-organizing network (SOM) that consists of input layer, and Kohonen or clustering layer.

Finally the categorized data is obtained from the SOM. From this obtained categorized data, must link and cannot link constraints are derived. The data points in a cluster are considered as must link constraint and data points outside the clusters are considered as cannot link constraints. These constraints are used in the constraints checking module of constrained K-Means algorithm.

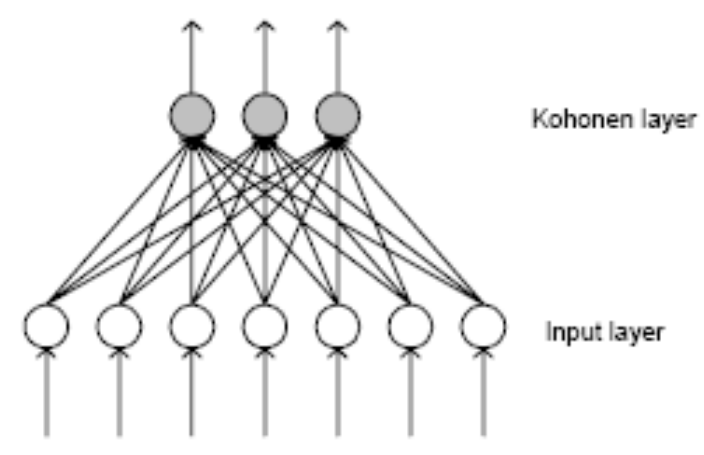

Figure 1: Architecture of Self-Organizing Map

\section{EXPERIMENTAL RESULTS}

The experiment is performed based on DBLP dataset, each of them uses a snapshot of co-authorship network at a certain year and predicts the venues in which a researcher will participate in the next year. DBLP data was downloaded in July, 2009. It contains 788,259 authors, $1,226,412$ publications and 3,490 venues. We extract snapshots of co-authorship network in the years of 2005. The snapshots are created based on the publications from the considered year backwards, e.g. 2005 snapshot takes the co-authorship network of publications published in 2005 or earlier. Each snapshot is presented as a weighted un-directed graph, where nodes are researchers and there is an edge between two researchers if they co-authored at least one publication. The edges are weighted by the number of co-authored publications. The 2005 network contains 478,108 nodes and 1,427,196 edges. We run the density-based clustering algorithm [5] on each network.

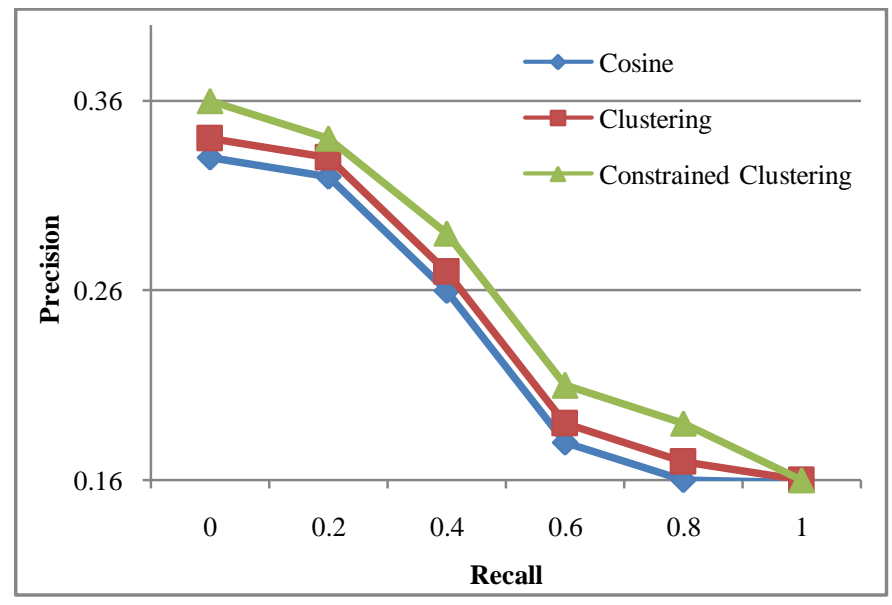

Figure 2: Performance Comparison of the Techniques based on Precision

Figure 2 shows the performance comparison of the techniques based on precision and recall. The precision-recall curves for 2005 and 2006 test cases are given in Figure 2. Clearly, constrained clustering method performs better than traditional Cosine $\mathrm{CF}$ and clustering technique.

The widely used technique for evaluating recommender systems is leave-one-out. This technique involves hiding one rating from the test set and trying to to predict it with a certain algorithm. Then the predicted rating is compared with the real rating and the difference in absolute value is the prediction error [9]. We use leave-one-out technique and employ the Mean Absolute Error (MAE) to measure the quality of the prediction of our approach in comparison with other collaborative methods and trust-based recommendation methods. Formally, MAE is defined as follows:

$$
R(r, v)=\frac{\sum_{i, j}\left|p_{i, j}-r_{i, j}\right|}{n}
$$

Where $\mathrm{n}$ is the number of ratings over all the test cases, $\mathrm{p}_{\mathrm{i}, \mathrm{j}}$ is the prediction rating of user $i$ on item $j$ and $r_{i, j}$ is the actual rating. We also use the coverage to measure the percentage of the time that an algorithm is able to successfully make a recommendation. Normally, this means that the algorithm was 
able to make some recommendations. A successful recommendation is defined as the prediction that the algorithm can make for a rating in the test set.

Table 1 shows the MAE comparison for the constrained clustering and the existing clustering technique. The proposed approach has very less MAE when compared to the existing technique.

Table 1: Mean Absolute Error Value Obtained for the Three Clusters

\begin{tabular}{|c|c|c|}
\hline & $\begin{array}{c}\text { Clustering } \\
\text { Collaborative } \\
\text { Filtering } \\
\text { Recommendation }\end{array}$ & $\begin{array}{c}\text { Constrained } \\
\text { Clustering } \\
\text { Collaborative } \\
\text { Filtering } \\
\text { Recommendation }\end{array}$ \\
\hline Cluster 1 & 0.3654 & 0.2000 \\
\hline Cluster 2 & 0.4542 & 0.2464 \\
\hline Cluster 3 & 0.3095 & 0.1943 \\
\hline
\end{tabular}

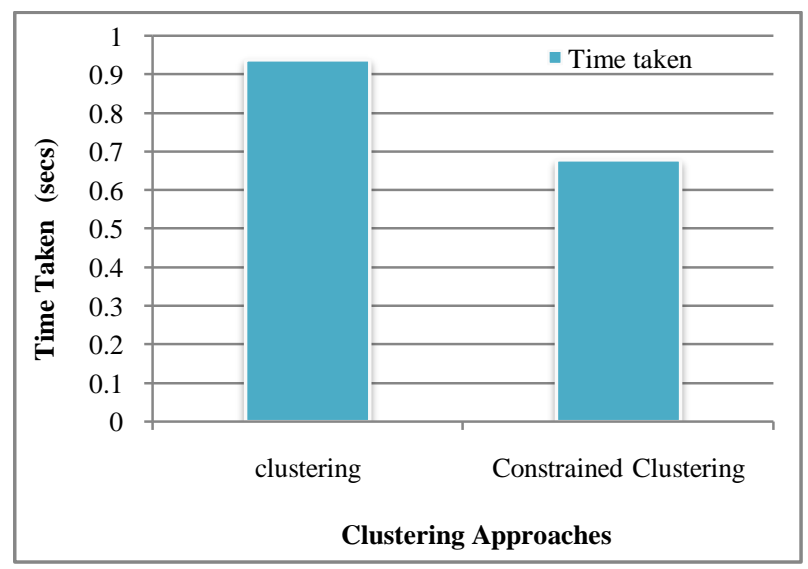

Figure 3: Performance Comparison based on Time Taken for Clustering

Figure 3 shows the time taken for the clustering technique. From the figure, it is clear that the proposed approach takes very less time when compared with the existing technique.

\section{CONCLUSIONS AND FUTURE WORK}

An efficient clustering approach to collaborative filtering recommendation technique is proposed in this paper. Instead of using ratings data, this proposed approach uses social relationship between users to identify their neighborhoods. Clustering plays an important role in the recommendation system performance. A complex network clustering technique is applied on the social network of users to find the groups of similar users. In this proposed approach, constrained clustering technique is used. After that, the traditional $\mathrm{CF}$ algorithms can be used to efficiently generate the recommendations. The experiments are conducted with DBLP data sets. The performance of the proposed approach is evaluated based on certain parametric standards like precision, recall, Mean Absolute Error and time taken for classification.
It is observed from the experimental observation that the proposed constrained clustering approach for collaborative filtering recommendation outperforms the existing clustering approach for collaborative filtering recommendation. The future work of this research would be to use Association rule mining for effective recommendation system.

\section{REFERENCES}

[1] Adomavicius, G. and Tuzhilin, A. "Toward the next generation of recommender systems: A survey of the state-of-the-art and possible extensions", Knowledge and Data Engineering, IEEE Transactions on, 17(6):734- 749, 2005.

[2] Anglade, A., Tiemann, M., and Vignoli, F. "Complex network theoretic clustering for identifying groups of similar listeners in p2p systems", In RecSys '07: Proceedings of the 2007 ACM conference on Recommender systems, pages 41-48, New York, NY, USA. ACM, 2007.

[3] Backstrom, L., Huttenlocher, D., Kleinberg, J., and Lan, X. "Group formation in large social networks: membership, growth, and evolution", In KDD '06: Proceedings of the 12th ACM SIGKDD international conference on Knowledge discovery and data mining, pages 44-54, New York, NY, USA. ACM, 2006. [Breese et al., 1998]

[4] Burke, R. "Hybrid recommender systems: Survey and experiments", User Modeling and User-Adapted Interaction, 12(4):331-370, 2002.

[5] Clauset, A., Newman, M. E. J., and Moore, C. "Finding community structure in very large networks” Phys. Rev. E, 70(6):066111, 2004.

[6] George, T. and Merugu, S. "A scalable collaborative filtering framework based on co-clustering", In ICDM '05: Proceedings of the Fifth IEEE International Conference on Data Mining, pages 625-628, Washington, DC, USA. IEEE Computer Society, 2005.

[7] SongJie Gong, Hongwu Ye and Ping Su, "A Peer-to-Peer based Distributed Collaborative Filtering Architecture", International Joint Conference on Artificial Intelligence, 2009.

[8] Harper, F. M., Sen, S., and Frankowski, D. "Supporting social recommendations with activity-balanced clustering", In RecSys '07: Proceedings of the 2007 ACM conference on Recommender systems, pages 165-168, New York, NY, USA. ACM, 2007.

[9] Herlocker, J. L., Konstan, J. A., Terveen, L. G., and Riedl, J. T. "Evaluating collaborative filtering recommender systems", ACM Trans. Inf. Syst., 22(1):5-53, 2004.

[10] Hofmann, T. "Latent semantic models for collaborative filtering", ACM Trans. Inf. Syst., 22(1):89-115, 2004.

[11] Guanfeng Liu, Yan Wang, Mehmet A. Orgun and Ee-Peng Lim, "A Heuristic Algorithm for Trust-Oriented Service Provider Selection in Complex Social Networks", IEEE International Conference on Services Computing, 2010.

[12] Richard Nock and Frank Nielsen, "Improving Clustering Algorithms through Constrained Convex Optimization", Proceedings of the 17th International Conference on Pattern Recognition (ICPR'04), 2004.

[13] Koren, Y., Bell, R., and Volinsky, C. Matrix factorization techniques for recommender systems. Computer, 42(8):30-37, 2009.

[14] Breese, J. S., Heckerman, D., and Kadie, C "Empirical analysis of predictive algorithms for collaborative filtering", In Proceedings of the14th Annual Conference on Uncertainty in Artificial Intelligence (UAI98), pages 43-52, 1998.

[15] Ungar, L. and Foster, D. "Clustering methods for collaborative filtering", In Proceedings of the Workshop on Recommendation Systems. AAAI Press, Menlo Park California, 1998.

[16] Wang, F., Ma, S., Yang, L., and Li, T. (2006). Recommendation on item graphs. In ICDM '06: Proceedings of the Sixth International Conference on Data Mining, pages 1119-1123, Washington, DC, USA. IEEE Computer Society, 2006.

[17] Zhang, J. and Pu, P. "A recursive prediction algorithm for collaborative filtering recommender systems”. In RecSys '07: Proceedings of the 2007 ACM conference on Recommender systems, pages 57-64, New York, NY, USA. ACM, 2007.

[18] K. Wagstaff, C. Cardie, S. Rogers and S. Schroedl, "Constrained Kmeans clustering with background knowledge", in: Proc. Of 18th Int. Conf. on Machine Learning ICML'01, p. 577 - 584. 